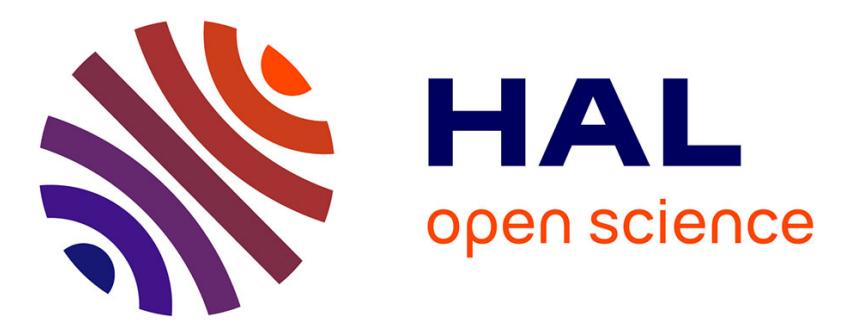

\title{
Scarce state transition turbo decoding based on re-encoding combined with a dummy insertion
}

Haisheng Liu, Christophe Jego, Emmanuel Boutillon, Michel Jezequel, Jean-Philippe Diguet

\section{To cite this version:}

Haisheng Liu, Christophe Jego, Emmanuel Boutillon, Michel Jezequel, Jean-Philippe Diguet. Scarce state transition turbo decoding based on re-encoding combined with a dummy insertion. Electronics Letters, 2009, 45 (16), pp. 846-848. hal-00417714

\section{HAL Id: hal-00417714 https://hal.science/hal-00417714}

Submitted on 16 Sep 2009

HAL is a multi-disciplinary open access archive for the deposit and dissemination of scientific research documents, whether they are published or not. The documents may come from teaching and research institutions in France or abroad, or from public or private research centers.
L'archive ouverte pluridisciplinaire HAL, est destinée au dépôt et à la diffusion de documents scientifiques de niveau recherche, publiés ou non, émanant des établissements d'enseignement et de recherche français ou étrangers, des laboratoires publics ou privés. 


\title{
Scarce state transition turbo decoding based on re-encoding combined with a dummy insertion
}

\author{
H. Liu, C. Jégo, E. Boutillon, J-Ph. Diguet, and M. Jézéquel
}

An efficient way to reduce the dynamic power dissipation in the turbo decoder is presented. This technique is based on a dynamic re-encoding of the received messages. The idea is to decrease the state transition activity of the trellis-based algorithms by replacing the classical direct decoding of the random noisy codewords by an equivalent decoding of an almost "all zero" codewords in order to keep the survivor path on the "zero path".

Introduction: Forward Error Correction (FEC) is one of the most powerful techniques that improve performance of digital communication. Currently, the turbo code family [1] is considered to be an efficient coding scheme for channel coding. They are particularly attractive for mobile communication systems and have been included in the specifications for both the UMTS and CDMA2000 third-generation cellular standards. In mobile communication systems, the adoption of turbo codes has consistently increased the share of channel decoding in the total receiver energy budget from around $30 \%$ to almost $50 \%$. This means that the channel decoder is becoming the main energy bottleneck in the mixed-signal receiver [2]. For convolutional turbo codes, the decoding is performed using the BCJR algorithm, also known as the MAP algorithm. In practice, an approximation of the log-domain derivation of the algorithm (max-log-MAP) is used.

This letter describes a technique based on re-encoding to decrease the state transition activity in the max-log-MAP algorithm and consequently the dynamic 
power dissipation in the turbo decoder. The idea of re-encoding a received codeword was first introduced in the Welch-Berlekamp (WB) algorithm [3] for the decoding of algebraic block codes such as Reed-Solomon (RS) codes. The WB algorithm concentrates on finding a codeword by translating an error pattern into another more likely error pattern in the same coset. A first error pattern is obtained by re-encoding. It corresponds to the error pattern which assumes that all message symbols are correct and that all errors occurred in the check symbols. The same year, a scarce state transition (SST) approach was introduced in [4] to reduce the power consumption of the Viterbi decoding systems for convolutional codes. The received data is precoded before decoding by a conventional Viterbi decoder. The similar re-encoding technique was further extended to the Soft Output Viterbi Algorithm (SOVA) for the turbo code decoding [5]. To our best knowledge, no previous study was done to apply this technique for decoding turbo codes based on the maxlog-MAP algorithm.

Re-encoding principle: This technique can be introduced by using the state space representation

$$
\left\{\begin{array}{c}
\mathbf{X}_{\mathbf{k}+1}=[\mathbf{A}] \times \mathbf{X}_{\mathbf{k}}+[\mathbf{B}] \times D_{k} \\
\mathbf{V}_{\mathbf{k}}=[\mathbf{C}] \times \mathbf{X}_{\mathbf{k}}+[\mathbf{D}] \times D_{k}
\end{array}\right.
$$

of the encoder [6]. The mapping between the information sequence $D_{k}$ and the code sequence $V_{k}$ at time $k$ depends on the state of the encoder $X_{k}$. Let us consider a Recursive Systematic Convolutional (RCS) code defined by the generator polynomial $\mathrm{G}=(5,7)_{\text {octal. }}$ The corresponding state matrices can be expressed as: $[\mathbf{A}]=\left(\begin{array}{ll}0 & 1 \\ 1 & 0\end{array}\right), \quad[\mathbf{B}]=\left(\begin{array}{l}1 \\ 0\end{array}\right), \quad[\mathbf{C}]=\left(\begin{array}{ll}0 & 0 \\ 1 & 0\end{array}\right)$ and $\quad[\mathbf{D}]=\left(\begin{array}{l}1 \\ 1\end{array}\right)$ and 
the code sequence is $\mathbf{V}_{k}=\left(\begin{array}{l}D_{k} \\ R_{k}\end{array}\right)$ where $D_{k}$ represent the systematic part of the codeword and $R_{k}$ the redundancy. The state space representation of the encoder becomes:

$$
\begin{aligned}
& \mathbf{X}_{\mathrm{k}+1}=\left(\begin{array}{l}
\mathrm{X}_{\mathrm{k}+1}^{1} \\
\mathrm{X}_{\mathrm{k}+1}^{2}
\end{array}\right)=\left(\begin{array}{ll}
0 & 1 \\
1 & 0
\end{array}\right) \times\left(\begin{array}{l}
X_{\mathrm{k}}^{1} \\
\mathrm{X}_{\mathrm{k}}^{2}
\end{array}\right)+\left(\begin{array}{l}
1 \\
0
\end{array}\right) \mathrm{D}_{\mathrm{k}} \\
& \mathbf{V}_{\mathbf{k}}=\left(\begin{array}{l}
\mathrm{D}_{\mathrm{k}} \\
\mathrm{R}_{\mathrm{k}}
\end{array}\right)=\left(\begin{array}{ll}
0 & 0 \\
1 & 0
\end{array}\right) \times\left(\begin{array}{l}
X_{\mathrm{k}}^{1} \\
\mathrm{X}_{\mathrm{k}}^{2}
\end{array}\right)+\left(\begin{array}{l}
1 \\
1
\end{array}\right) \mathrm{D}_{\mathrm{k}}
\end{aligned}
$$

Let $d_{k}=D_{k}+\varepsilon_{D_{k}}$ and $r_{k}=R_{k}+\varepsilon_{R_{k}}$ denote the soft bit sequences corresponding to the transmitted bit sequences $D_{k}$ and $R_{k}$, respectively. The variables $\varepsilon_{D_{k}}$ and $\varepsilon_{\mathrm{R}_{\mathrm{k}}}$ correspond to the noise error sequences added to the transmitted bit sequences.

$$
\mathbf{v}_{\mathbf{k}}=\left(\begin{array}{c}
\mathrm{d}_{\mathrm{k}} \\
\mathrm{r}_{\mathrm{k}}
\end{array}\right)=\left(\begin{array}{l}
\mathrm{D}_{\mathrm{k}} \\
\mathrm{R}_{\mathrm{k}}
\end{array}\right)+\left(\begin{array}{c}
\varepsilon_{\mathrm{D}_{\mathrm{k}}} \\
\varepsilon_{\mathrm{R}_{\mathrm{k}}}
\end{array}\right)
$$

Let $D^{h}{ }_{k}$ and $V_{k}^{h}$ denote the hard bit sequences corresponding to the soft bit sequences $d_{k}$ and $v_{k}$ respectively. A re-encoding step can be applied to the systematic part $D_{k}^{h}: \mathbf{V}_{\mathbf{k}}=[\mathbf{C}] \times \mathbf{X}_{\mathbf{k}}^{\prime}+[\mathbf{D}] \times \mathbf{D}_{\mathbf{k}}^{\mathbf{h}}$. We obtain

$$
\mathbf{V}_{k}^{\prime}=\left(\begin{array}{l}
\mathrm{D}_{\mathrm{k}}^{\prime} \\
\mathrm{R}_{\mathrm{k}}{ }^{\prime}
\end{array}\right)=\left(\begin{array}{ll}
0 & 0 \\
1 & 0
\end{array}\right) \cdot\left(\begin{array}{c}
\mathrm{x}^{\prime{ }_{k}} \\
\mathrm{x}^{\prime 2}{ }_{\mathrm{k}}
\end{array}\right)+\left(\begin{array}{l}
1 \\
1
\end{array}\right) \cdot \mathrm{D}^{\mathrm{h}}{ }_{\mathrm{k}}
$$

Finally, the two sequences $\mathrm{V}^{\mathrm{h}}{ }_{\mathrm{k}}$ and $\mathrm{V}^{\prime}{ }_{\mathrm{k}}$ are combined: $\mathbf{V}^{{ }^{\prime}{ }_{\mathbf{k}}}=\mathbf{V}^{\mathbf{h}}{ }_{\mathbf{k}}+\mathbf{V}_{\mathbf{k}}$. Using (4) and (5):

$$
\mathbf{V}^{\prime \prime}=\left(\begin{array}{l}
D^{\prime \prime}{ }_{k} \\
R^{\prime \prime}{ }_{k}
\end{array}\right)=\left(\begin{array}{l}
d_{k} \\
r_{k}
\end{array}\right)+\left(\begin{array}{c}
D_{k}^{\prime} \\
R_{k}^{\prime}
\end{array}\right)=\left(\begin{array}{c}
0 \\
R_{k}+\varepsilon_{R_{k}}+R_{k}^{\prime}
\end{array}\right)
$$

If we consider that the initial state of the encoder is equal to zero $\left(X_{0}=0\right)$ then the current state $X_{k}$ can be expressed as:

$$
\mathbf{X}_{\mathbf{k}}=\sum_{\mathbf{i}=1}^{\mathbf{k}}[\mathbf{A}]^{\mathbf{i}-1}[\mathbf{B}] . \mathbf{D}_{\mathbf{k}-\mathbf{i}}
$$


Similarly, if the initial state of the re-encoder is equal to zero $\left(X_{0}^{\prime}=0\right)$ then the current state $\mathbf{X}_{\mathrm{k}}{ }_{\mathrm{k}}$ can be expressed as:

$$
\mathbf{X}_{\mathbf{k}}{ }_{\mathbf{k}}=\sum_{\mathbf{i}=1}^{\mathbf{k}}[\mathbf{A}]^{\mathbf{i}-1}[\mathbf{B}] \cdot \mathbf{d}_{\mathbf{k}-\mathbf{i}}=\sum_{\mathbf{i}=1}^{\mathbf{k}}[\mathbf{A}]^{\mathbf{i}-1}[\mathbf{B}] \cdot \mathbf{D}_{\mathbf{k}-\mathbf{i}}+\sum_{\mathbf{i}=1}^{\mathbf{k}}[\mathbf{A}]^{\mathbf{i}-1}[\mathbf{B}] \cdot \varepsilon_{\mathbf{D}_{\mathbf{k}-\mathbf{i}}}
$$

A new expression for equation (6) can be thus obtained from (7) and (8):

$$
\mathbf{V}^{\prime \prime}{ }_{k}=\left(\begin{array}{c}
0 \\
X^{1}{ }_{k}+D_{k}+\varepsilon_{R_{k}}+X^{\prime 1}{ }_{k}+D_{k}+\varepsilon_{D_{k}}
\end{array}\right)=\left(\begin{array}{c}
0 \\
\varepsilon_{R_{k}}+\varepsilon_{D_{k}}+\sum_{i=1}^{k-1}[A]^{i-1}[B] \varepsilon_{D_{k-i}}
\end{array}\right)
$$

It means that the sequence of the systematic part of $\mathrm{V}^{\prime \prime}{ }_{\mathrm{k}}$ is equal to zero. The value of the redundancy part of V"' ${ }_{k}$ at time $\mathrm{k}$ is equal to the sum of the noise with the accumulation of the noise for the systematic part from the initial state of the system. Note that this re-encoding technique can also be applied if the code is not systematic. However, a previous decoding step has to be applied to regenerate the information sequence as suggested in [4].

Iterative decoding of turbo codes based on re-encoding: The proposed turbo decoding scheme is composed of two systematic convolutional encoders and two max-log-MAP SISO decoders as shown in Figure 1. First, the APP sequence $z_{2}$ is added to the soft information sequence $d$ in the natural domain. In parallel, the APP interleaved sequence $\bar{z}_{1}$ is added to the soft interleaved information sequence $\bar{d}$ in the interleaved domain. Then, the sign-bits of the obtained sequences are only considered for the re-encoding step. The produced parity bit sequences $R_{i}$ are XORed with the sign-bits of the soft decision symbol sequences $r_{1}$ and $r_{2}$ in the natural and interleaved domains, respectively. The sign-bits of soft information sequences are thus replaced by sequences of zero. Traditional max-log-MAP algorithms are used 
to produced the APPs $z_{1}$ and $\bar{z}_{2}$. But, in order to obtain the correct values, the obtained APP sign-bits have to be XORed with the sign-bits of the soft information sequences $\mathrm{d}$ and $\overline{\mathrm{d}}$. After several half-iterations, the iterative process converges as in traditionally max-log-MAP decoder and the hard decisions are taken. The linearity of the code implies that the proposed approach has no impact on the BER performance. The transitions inside the trellis during the forward and the backward recursions of the max-log-MAP algorithm can thus considerably be reduced. Indeed, if the survivor path is maintained on the zero-path (the path that goes through the states 0 ) of the trellis then most of the state transitions can be avoided. Note that the metric value of state 0 is turned into a zero by subtracting the corresponding transition metric from the other transition metrics.

Dummy insertion in the trellis: To verify the efficiency of the re-encoding technique for turbo decoding, a study of the survivor paths has been done. Unfortunately, this study shows that the survivor paths do not always remain on the zero-path during a soft decoding process. Simulation results showed that the survivor path remains on the zero-path for $19 \%$ and $68 \%$ of the states at BERs of $10^{-1}$ and $10^{-6}$ if the re-encoding technique is applied. Actually, some survivor paths run along a particular periodic path that just depends on the constraint length of the code. So, it is possible to bring the survivor path back to the zero-path. This technique is called "dummy insertion" in this paper. It consists in adding some bits to the sign-bits of the transmitted soft information sequence $d$ for removing the cyclic binary patterns in the parity bit sequence. These dummy bits are then removed after the decoding process thanks to a FIFO memory and an Xor operation. If the 
re-encoding technique is combined with the dummy insertion approach then the survivor path remains on the zero-path for $40 \%$ and $89 \%$ of the states at BERs of $10^{-1}$ and $10^{-6}$, respectively. Reducing the state transition activities, the scarce state transition turbo decoding reduces the dynamic power consumption. The principle of circularity is generally adopted to ensure the continuity of the states at both ends of information blocks. This process is known as trellis closing. For RCS codes, the addition of extra bits to the initial message enables to transmit the final state. Another well-known technique consists of adopting a circular convolutional code which guarantees that the initial and the final states are identical. The scarce state transition turbo decoding based on re-encoding technique combined with a dummy insertion approach has to be extended to the Recursive Systematic Circular Convolutional (RCCS) codes. A solution is to set the state zero for the initial and the final states. This process of closing requires forcing the encoder to the final state zero by changing the last bits. These bits ensuring the closing of the trellis are not decoded by the receiver.

Re-encoding combined with a dummy insertion for the turbo code decoding: The proposed architecture for the two domains (natural and interleaved) of the turbo decoding process is detailed in Figure 2. The main innovation compared to the architecture presented in Figure 1 is about the dummy insertion process. The dummy block contains logic elements (Flip-Flop, Xor and And) for detecting the binary pattern but also for constructing the $D_{u}$ dummy signal. Finally, an Xor logic gate is necessary to add the dummy-bits to the sign-bit of d. It means that the additional hardware cost of the re-encoding process is 
very low. It has no significant impact on the total power dissipation. As expected, the performance in terms of Bit Error Rate (BER) of the proposed decoding of convolutional turbo codes is strictly identical with the traditional turbo decoding.

Conclusion: A scarce state transition turbo decoding based on re-encoding technique combined with a dummy insertion approach has been presented. This technique helps in reducing the state transition activity of the max-logMAP algorithm by maintaining the survivor path on the zero-path of the trellis. Simulation results showed that the state transition activity of the turbo decoding process is thus significantly reduced with no performance degradation. The design of a turbo decoder based on the proposed technique is under study. The objective is to measure the total power dissipation of the circuit.

\section{References}

1 Berrou C., Glavieux A., Thitimajshima P.: 'Near Shannon limit error correcting coding and decoding: Turbo Codes', IEEE International Conference on Communication ICC93, vol. 2/3, May 1993.

2 B. Bougard, "Cross-layer energy management in broadband wireless transceivers," PhD dissertation, March 2006.

3 L.R.Welch and E.R. Berlekamp, "Error Correction for Algebraic Block Codes," US Patent 4,633,470, 1986.

4 S. Kubota, K. Ohtani, and S. Kato, "High-speed and high-coding-gain Viterbi decoder with low power consumption employing SST (scarce state transition) scheme", Electronics Letters, vol. 22, issue 9, pp. 491-493, 24 April 1986.

5 Lang Lin, Chi Ying Tsui and R.S. Cheng, "Low power soft output Viterbi decoder scheme for turbo code decoding", IEEE Intern. Symp. On Circuits and Systems, ISCAS07, Hong Kong, Vol.2, June 1997, pp. 1369-1372

6. C. Weiss, C. Bettstetter, S. Riedel and D. J. Costello, "Turbo decoding with tail-biting trellises”, In URSI Int. Sig. Sys. and Elec., 1998. 
Authors' affiliations:

H. Liu, C. Jégo and M. Jézéquel

(Institut Telecom/Telecom Bretagne, CNRS Lab-STICC, Brest, France)

J-P. Diguet and E. Boutillon

(Université de Bretagne Sud, CNRS Lab-STICC, Lorient, France)

\section{Figure captions:}

Fig. 1 Block diagram of a turbo decoder with re-encoding technique

Fig. 2 Block diagram of the scarce state transition SISO decoder 
Figure 1

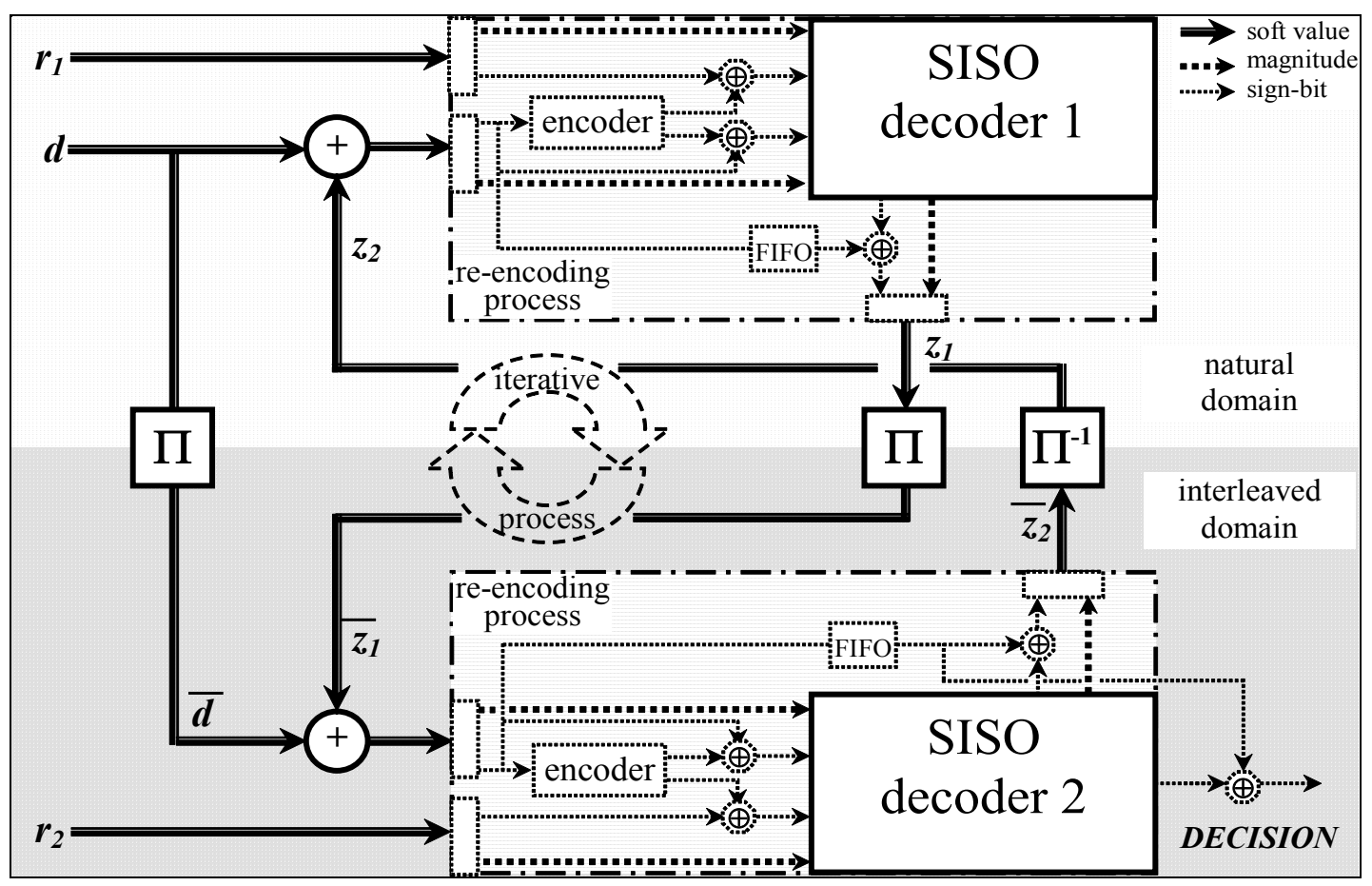

Figure 2

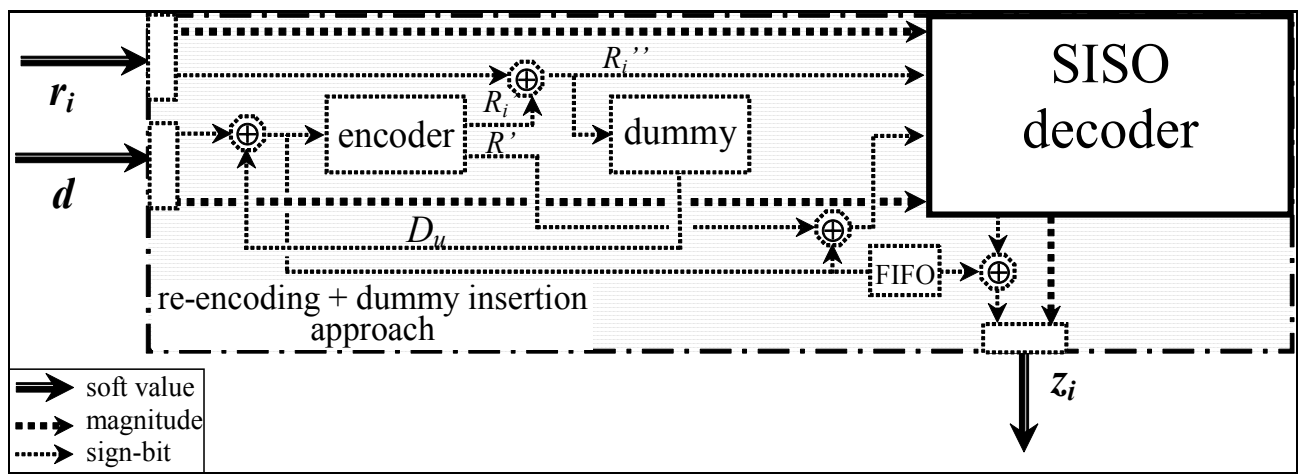

